\title{
DOMINANCE MEASURING METHODS FOR THE SELECTION OF CLEANING SERVICES IN A EUROPEAN UNDERGROUND TRANSPORTATION COMPANY*
}

\author{
Antonio Jiménez-Martín ${ }^{1}$, Alfonso Mateos $^{1}$ And Juan A. Fernández del Pozo ${ }^{1}$
}

\begin{abstract}
Dominance measuring methods are a recent approach for dealing with complex decisionmaking problems with imprecise, incomplete or partial information within multi-attribute value/utility theory. These methods compute pairwise dominance values and exploit the information included in the dominance matrix in different ways to derive measures of dominance intensity to rank the alternatives under consideration. We review dominance measuring methods proposed in the literature, describing how their possible drawbacks have been progressively overcome, and comparing their performance with other existing approaches, like surrogate weighting methods, the adaptation of classical decision rules to encompass an imprecise decision context, SMAA or Sarabando and Dias' method. An example of the selection of cleaning services in a European underground transportation company is used to illustrate dominance measuring methods in a real complex decision-making problem.
\end{abstract}

Mathematics Subject Classification. 91B06, 91B16.

Received May 11, 2015. Accepted May 20, 2016.

\section{INTRODUCTION}

The additive model is widely used within multi-attribute value/utility theory (MAVT/MAUT) to rank alternatives in complex decision-making problems. It is considered a valid approach in many practical situations for the reasons described in $[23,29]$.

However, the information available in most real complex decision-making problems is not precise. Inputs are often described within prescribed bounds or as just satisfying certain relations. Different authors refer to this situation as decision-making with imprecise information, incomplete information or partial information [24,25].

Several reasons are given in the literature that justify why a decision-maker (DM) may wish to provide imprecise information [28,32]. For example, performances that reflect social or environmental impacts may be intangible or non-monetary, and performances may be taken from statistics or measurements. Besides, a DM might prefer not to reveal his/her preferences in public or could feel more comfortable providing a scale to represent attribute importance. Moreover, the decision could be taken in a group decision-making situation, where a negotiation process usually outputs imprecise information.

Keywords. Dominance measuring methods, imprecise information, multi-attribute value/utility theory.

* The paper was supported by the Spanish Ministry of Economy and Competitiveness project MTM2014-56949-C3-2-R.

1 Departamento de Inteligencia Artificial, Universidad Politécnica de Madrid, Campus de Montegancedo S/N, 28660 Boadilla del Monte, Spain. \{antonio.jimenez, alfonso.mateos, juan.fdezpozo.salamanca\}@upm.es 
Many papers on MAVT/MAUT have dealt with imprecise information. Sarabando and Dias [27] provided a brief overview of approaches proposed by different authors within the MAVT/MAUT framework to deal with imprecise information.

For instance, surrogate weighting methods (SWMs) deal with ranked attribute weights to output a best alternative and/or ranking of alternatives [4,31]. A weight vector is selected from a set of admissible weights to represent the set, which is then used to evaluate the alternatives by means of the multi-attribute value model. Commonly used SWMs are rank sum weights $(R S)$, rank reciprocal weights $(R C)$, rank-order centroid weights $(R O C)$, and equal weights $(E W)$.

The stochastic multicriteria acceptability analysis (SMAA) method was proposed to provide support when the weight information is missing [12]. The SMAA-2 method [11] extends the analysis to the sets of weight vectors for any rank from best to worst for each decision alternative and can be used to identify good compromise alternatives, whereas SMAA-O [13] is a variant of SMAA for problems in which criteria are measured on ordinal scales.

Another option described in the literature for dealing with imprecision is based on the concepts of pairwise and absolute dominance.

The use of absolute dominance values is exemplified by the modification of four classical decision rules to encompass an imprecise decision context concerning weights and component values/utilities [22, 26]: the maximax, in which each alternative is rated on the basis of its maximum guaranteed value; the maximin, based on its minimum guaranteed value; the minimax regret rule, based on the maximum loss of value with respect to a better alternative; and the central value rule, based on the midpoint of the range of possible performances.

Regarding pairwise dominance, Eum et al. $[5,14,21]$ provided linear programming characterizations of dominance and potential optimality for alternatives when information about values and/or weights is incomplete amd extended the approach to hierarchical structures [21]. Later, the concepts of weak potential optimality and strong potential optimality were developed in [14]. Besides, dominance and potential optimality with imprecision in weights, utilities and alternative performances is considered in [15].

A recent approach for dealing with imprecise information is to compute different measures of dominance to derive a ranking of alternatives. They are known as dominance measuring methods (DMMs). DMMs are based on the computation of a dominance matrix, $D$, including pairwise dominance values, which are exploited in different ways to derive measures of dominance to rank the alternatives under consideration.

In Section 1, we give an overview of the DMMs proposed in the literature and analyze their performance against the other existing approaches described above. An example concerning the selection of cleaning services in a European underground transportation company illustrates DMMs in Section 2. Finally, some conclusions are provided in Section 3.

\section{Dominance MeASURING MEthods}

We consider a decision-making problem with $m$ alternatives $\left\{A_{1}, \ldots, A_{m}\right\}$ and $n$ attributes $\left\{X_{1}, \ldots, X_{n}\right\}$, then the functional form of the additive model is

$$
v\left(A_{i}\right)=\sum_{j=1}^{n} w_{j} v_{j}\left(x_{i j}\right),
$$

where $x_{i j}$ is the performance over attribute $X_{j}$ for alternative $A_{i}$, and $v_{j}$ and $w_{j}$ are the component value/utility function and the weight for attribute $X_{j}$, respectively. Note that $\sum_{j=1}^{n} w_{j}=1$ and $w_{j} \geq 0$.

The alternative performances could be precise or described under uncertainty by means of uniformly distributed intervals, i.e.,

$$
x_{i j}^{L} \leq x_{i j} \leq x_{i j}^{U}, i=1, \ldots, m, j=1, \ldots, n .
$$

Besides, imprecision could be also considered regarding DM preferences, both when assessing DM single attribute utilities, which represent DM preferences concerning the possible alternative performances, and weights, which represent the relative importance of criteria. 


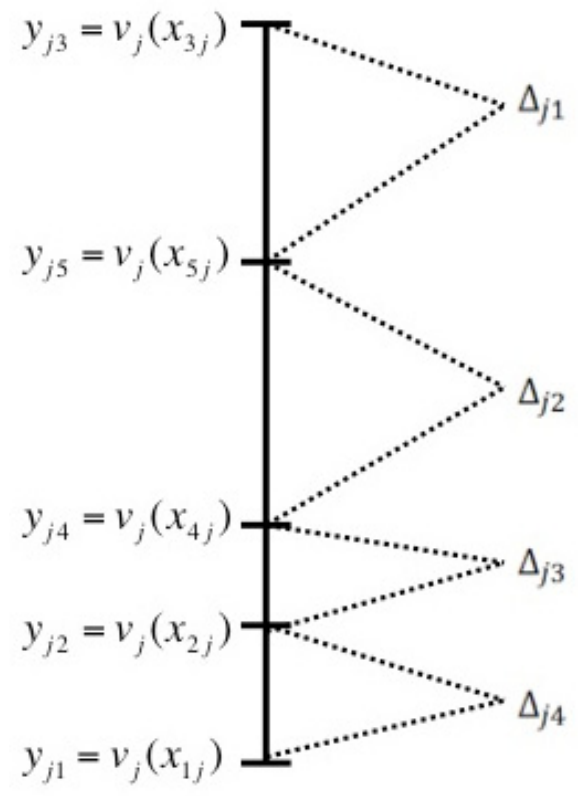

FiguRE 1. Ranking of alternatives and differences between consecutive alternatives for the attribute $X_{j}$.

In the first case, classes of component value/utility functions could be derived from the elicitation methods [6], and, consequently, the component value/utility associated with a specific performance would belong to an interval:

$$
v_{j}^{L}\left(x_{i j}\right) \leq v_{j}\left(x_{i j}\right) \leq v_{j}^{U}\left(x_{i j}\right), i=1, \ldots, m, j=1, \ldots, n .
$$

Alternatively, ordinal information about the component values/utilities of the alternatives could be considered $[2,28]$, i.e., the DM provides a ranking of the alternatives in each attribute. Moreover, rankings of the difference between the values of consecutive alternatives could be also taken into account for each attribute. For instance, the DM might consider $A_{3}$ to be the best of five alternatives for attribute $X_{j}$, followed by $A_{5}$, $A_{4}, A_{2}$ and $A_{1}\left(v_{j}\left(x_{3 j}\right) \geq v_{j}\left(x_{5 j}\right) \geq v_{j}\left(x_{4 j}\right) \geq v_{j}\left(x_{2 j}\right) \geq v_{j}\left(x_{1 j}\right)\right)$. Also, the differences between consecutive alternatives might be ranked $\Delta_{j 2} \geq \Delta_{j, 1} \geq \Delta_{j, 4} \geq \Delta_{j, 3}$, with $\Delta_{j, 2}=v_{j}\left(x_{5 j}\right)-v_{j}\left(x_{4 j}\right), \Delta_{j, 1}=v_{j}\left(x_{3 j}\right)-v_{j}\left(x_{5 j}\right)$, $\Delta_{j, 4}=v_{j}\left(x_{2 j}\right)-v_{j}\left(x_{1 j}\right)$ and $\Delta_{j, 3}=v_{j}\left(x_{4 j}\right)-v_{j}\left(x_{2 j}\right)$, as illustrated in Figure 1.

Imprecision concerning weights representing the relative importance of criteria could also be represented by intervals or by means of ordinal information. An example of an interval would be:

$$
w_{j}^{L} \leq w_{j} \leq w_{j}^{U}, j=1, \ldots, n,
$$

If we consider ordinal information about weights [2,27], the DM would provide an attribute importance ranking, arranged in descending order from the most to the least important attribute:

$$
\mathbf{w} \in W=\left\{\mathbf{w}=\left(w_{1}, \ldots, w_{n}\right) \mid w_{1} \geq w_{2} \geq \ldots \geq w_{n} \geq 0\right\}, \text { and } \sum_{j=1}^{n} w_{i}=1 .
$$

As mentioned before, dominance measuring methods (DMMs) are based on the computation of a dominance matrix, $D$, including pairwise dominance values, which are exploited in different ways to derive measures of dominance to rank the alternatives under consideration. 


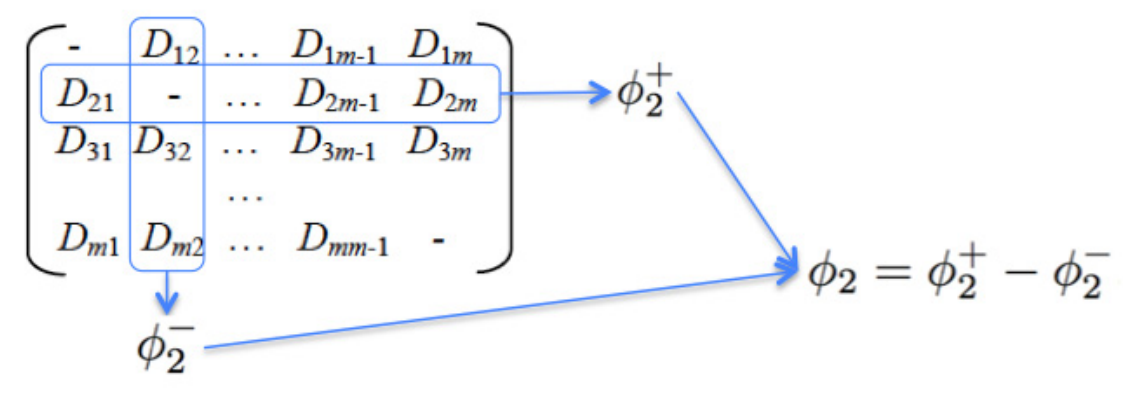

Figure 2. $A P 1$ and $A P 2$ methods.

A dominance matrix, $D$, can be defined as follows:

$$
D=\left(\begin{array}{ccccc}
- & D_{12} & \cdots & D_{1(m-1)} & D_{1 m} \\
D_{21} & - & \cdots & D_{2(m-1)} & D_{2 m} \\
D_{31} & D_{32} & \cdots & D_{3(m-1)} & D_{3 m} \\
\vdots & \vdots & \vdots & \vdots & \vdots \\
D_{m 1} & D_{m 2} & \cdots & D_{m(m-1)} & -
\end{array}\right)
$$

where

$$
\begin{array}{cc}
\begin{array}{c}
D_{k l}= \\
\text { s.t. }
\end{array} & \min \left\{\mathbf{w} \mathbf{v}_{k}-\mathbf{w v}_{l}\right\} \\
& \mathbf{v}_{k}=\left(v_{k 1}, \ldots, v_{k n}\right), \mathbf{v}_{l}=\left(v_{l 1}, \ldots, v_{l n}\right) \in V_{k l} \\
& \mathbf{w}=\left(w_{1}, \ldots, w_{n}\right) \in W,
\end{array}
$$

where $W$ and $V_{k l}$ define the feasible region for weights and values associated with the alternatives $A_{k}$ and $A_{l}$ for each attribute, respectively. They represent imprecise information.

Note that given two alternatives $A_{k}$ and $A_{l}$, alternative $A_{k}$ dominates $A_{l}$ if $D_{k l} \geq 0$, and there exists at least one $\mathbf{w}, \mathbf{v}_{k}$ and $\mathbf{v}_{l}$ such that the overall value of $A_{k}$ is strictly greater than that of $A_{l}$. This concept of dominance is called pairwise dominance.

The optimization problems to be solved to derive pairwise dominance values are different depending on how the imprecision concerning DM preferences is represented, but always we have a linear or quadratic programming problem that can be solved using the simplex method or Lemke's method, respectively.

The first DMM was proposed by Ahn and Park [3]. It was applicable when the imprecision concerning the DM preferences was represented by both intervals or ordinal information.

Ahn and Park compute a dominating measure $\phi_{k}^{+}=\sum_{j=1, j \neq k}^{m} D_{k j}$ and a dominated measure $\phi_{k}^{-}=\sum_{j=1, j \neq k}^{m} D_{j k}$ for each alternative $A_{k}$, and then derive a net dominance as $\phi_{k}=\phi_{k}^{+}-\phi_{k}^{-}$.

Ahn and Park proposed two ranking methods for these measures: ranking the alternatives according to either $\phi_{k}^{+}$or $\phi_{k}$ values (denoted as the AP1 and AP2 methods, respectively, see Fig. 2).

Two new $D M M s$ were proposed in $[16,18]$. The first one, DIM1, was based on the same idea as implemented by Ahn and Park. It also computed dominating and dominated measures but they were combined into a dominance intensity rather than a net dominance index, which was used as a measure of the strength of preference.

DIM1 was implemented as follows (see Fig. 3):

(1) Compute dominating indices for each alternative $A_{k}$ :

$$
D I_{k+}^{\text {row }}=\sum_{l=1, l \neq k, D_{k l}>0}^{m} D_{k l}, \quad D I_{k-}^{\text {row }}=\sum_{l=1, l \neq k, D_{k l}<0}^{m} D_{k l} .
$$




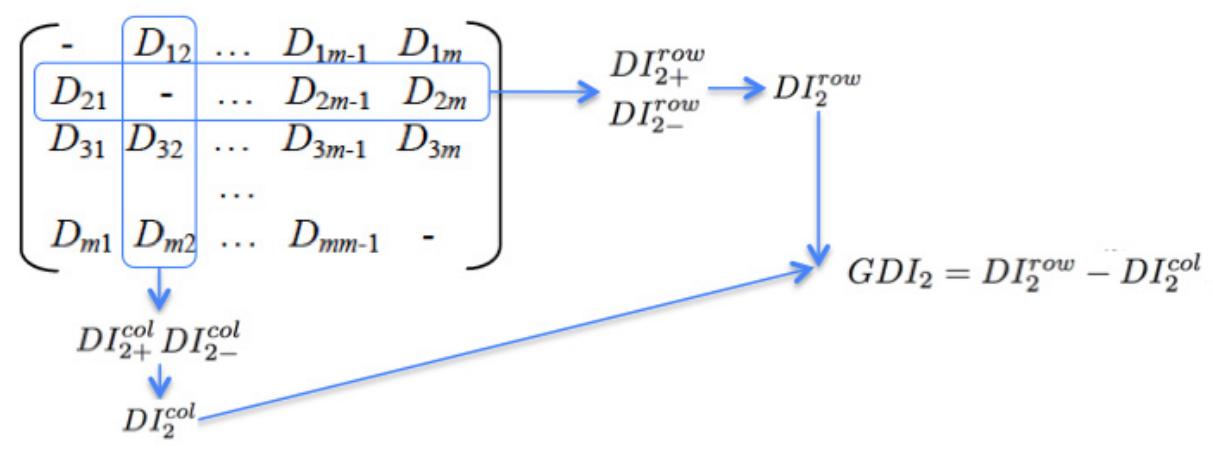

FiguRE 3. DIM1.

(2) Compute the dominating intensity $D I_{k}^{\text {row }}$ for each alternative $A_{k}$ :

$$
D I_{k}^{\text {row }}=\frac{D I_{k+}^{\text {row }}}{D I_{k+}^{\text {row }}-D I_{k-}^{\text {row }}} .
$$

(3) Compute dominated indices for each alternative $A_{k}$ :

$$
D I_{k+}^{\mathrm{col}}=\sum_{l=1, l \neq k, D_{l k}>0}^{m} D_{l k}, \quad D I_{k-}^{\mathrm{col}}=\sum_{l=1, l \neq k, D_{l k}<0}^{m} D_{l k} .
$$

(4) Compute the dominated intensity $D I_{k}^{\text {col }}$ for each alternative $A_{k}$ :

$$
D I_{k}^{\mathrm{col}}=\frac{D I_{k+}^{\mathrm{col}}}{D I_{k+}^{\mathrm{col}}-D I_{k-}^{\mathrm{col}}}
$$

(5) Calculate a global dominance intensity $(G D I)$ for each alternative $A_{k}$, i.e., $G D I_{k}=D I_{k}^{\text {row }}-D I_{k}^{\text {col }}, k=$ $1, \ldots, m$, and rank the alternatives accordingly.

DIM1 improves AP2 by reducing the duplicate information involved in the computations. However, DIM 1 has a drawback: the alternatives cannot be ranked if all the elements in $D$ are negative because, since $D I_{k+}^{\text {row }}=0$ and $D I_{k-}^{\text {row }}, \forall k$, their dominance intensity is zero in all cases. This drawback implies that DIM1 is not independent of irrelevant alternatives.

The second method, DIM2, overcomes this drawback. It derives a global dominance intensity index to rank alternatives on the basis that

$$
D_{k l} \leq \mathbf{w}^{T}\left(\mathbf{v}_{k}-\mathbf{v}_{l}\right) \leq-D_{l k}, \forall \mathbf{w} \in W, \mathbf{v}_{k}, \mathbf{v}_{l} \in V_{k l} .
$$

DIM2 was implemented as follows (see Fig. 4):

(1) If $D_{k l} \geq 0$, then alternative $A_{k}$ dominates $A_{l}$, and the dominance intensity of $A_{k}$ over $A_{l}\left(D I_{k l}\right)$ is 1 , i.e., $D I_{k l}=1$.

Else $\left(D_{k l}<0\right)$ :

- If $D_{l k} \geq 0$, then alternative $A_{l}$ dominates $A_{k}$, and $D I_{k l}=0$.

- Else $\left(D_{l k}<0\right), D I_{k l}=\frac{-D_{l k}}{-D_{l k}-D_{k l}}$.

(2) Calculate a global dominance intensity $(G D I)$ for each alternative $A_{k}$, i.e. $G D I_{k}=\sum_{l=1, l \neq k}^{m} D I_{k l}$, and rank the alternatives accordingly. 


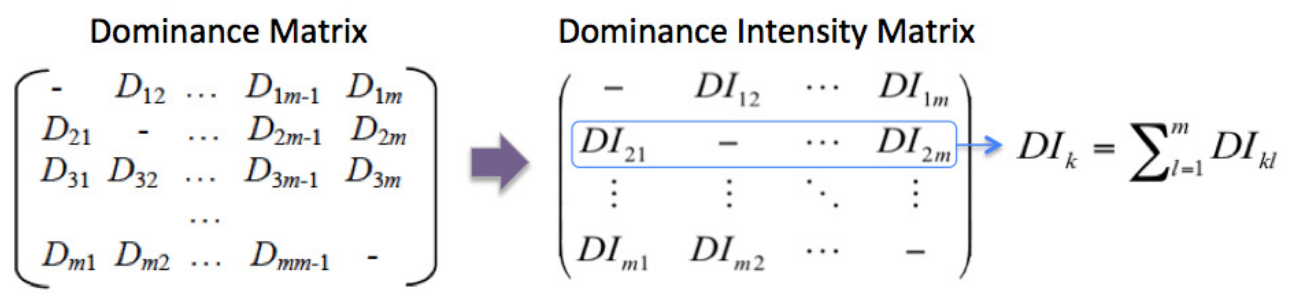

FiguRE 4. DIM2.

Simulation studies were carried out in [18] to compare the DIM1 and DIM2 methods with other existing approaches (the above modification of four classical decision rules, SMAA [12], SMAA-2 [11] and the AP1 and $A P 2$ methods) when the imprecision concerning the inputs is represented by value intervals.

We use two measures of efficacy: the hit ratio and the rank-order correlation $[3,4]$. The hit ratio is the proportion of all cases in which the method selects the same best alternative as in the TRUE ranking. Rankorder correlation indicates how similar the overall alternative ranking structures are in the TRUE ranking and in the ranking derived from the method. It is calculated using Kendall's $\tau$ [33]:

$$
\tau=1-\frac{2 \times(\text { number of pairwise preference violations })}{\text { total number of pair preferences }}=\frac{S}{m(m-1) / 2},
$$

where $S$ is the difference between the number of concordant (equally ordered) and discordant (differently ordered) pairs and $m$ is the total number of alternatives.

The results of simulation studies showed that DIM2 performs better than the AP1 method and the adaptation of classical decision rules. Although SMAA-2 slightly outperforms DIM2, DIM2 is applicable when incomplete information about weights is expressed not just as weight intervals but also as weights satisfying linear or nonlinear constraints, weights represented by fuzzy numbers or weights fitting normal probability distributions.

The performance of DIM1 and DIM2 was also compared in [20] with other existing approaches (surrogate weighting methods, modified classical decision rules and the AP1 and AP2 methods) when ordinal information represents imprecision concerning weights. As regards average hit ratios, DIM2 and ROC outperform the other methods and, according to the paired-samples t-test, there is no significant difference between the two. However, $R O C$ can be only applied when ordinal relations regarding attribute weights are provided.

Despite its good performance, we later identified a drawback associated with DIM2. It did not take into account the size of the intervals $\left[D_{k l},-D_{l k}\right]$ and how far they were from 0 . Figure 5 illustrates the above situations in which different intervals lead to the same dominance intensities, $D_{k l}$. A new DMM, DIM3, was proposed in $[17,19]$ aimed at overcoming these drawbacks. It was based on DIM2 and, specifically, on the fact that $\mathbf{w}^{T}\left(\mathbf{v}_{k}-\mathbf{v}_{l}\right) \in\left[D_{k l},-D_{l k}\right], \forall \mathbf{w} \in W, \mathbf{v}_{k}, \mathbf{v}_{l} \in V_{k l}$, but DIM3 incorporates the distance from the interval $\left[D_{k l},-D_{l k}\right]$ to 0 to derive a dominance intensity measure to rank the alternatives under consideration:

(1) If $D_{k l} \geq 0$, then alternative $A_{k}$ dominates $A_{l}$, and the dominance intensity of $A_{k}$ over $A_{l}$ is $D I_{k l}=$ $d\left(\left[D_{k l},-D_{l k}\right], 0\right)$.

Else $\left(D_{k l}<0\right)$ :

- If $D_{l k} \geq 0$, then $A_{l}$ dominates $A_{k}$, and $D I_{k l}=-d\left(\left[D_{k l},-D_{l k}\right], 0\right)$.

- Else $\left(D_{l k}<0\right)$,

$$
D I_{k l}=\left[\frac{-D_{l k}}{-D_{l k}-D_{k l}}-\frac{-D_{k l}}{-D_{l k}-D_{k l}}\right] \times d\left(\left[D_{k l},-D_{l k}\right], 0\right) .
$$

(2) Calculate a global dominance intensity $(G D I)$ for each alternative $A_{k}$, i.e., $G D I_{k}=\sum_{l=1, l \neq k}^{m} D I_{k l}$, and rank the alternatives accordingly. 


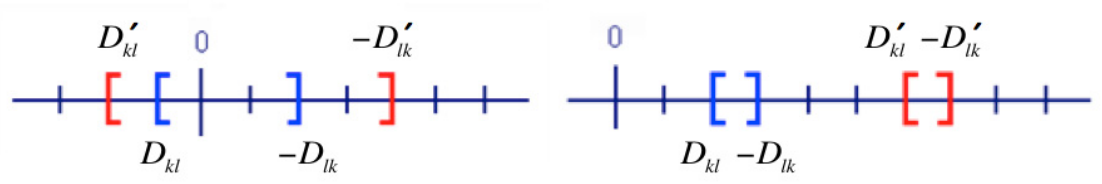

FiguRE 5. Drawbacks of DIM2.

New extensions of DIM2 and DIM3 were proposed in [1]. The first extension weights the dominance intensities derived in DIM2 and DIM3 according to the distance between the central weight vector $\left(w_{j}^{c}\right)$ and the weight vector $\left(w_{j}^{* l k}\right)$ associated with the optimal $D_{l k}$ to solve the corresponding optimization problem. Note that the central weight vector is composed of the midpoints of the different weight intervals in the case of interval weights.

The aim of these extensions is to attach more importance to weight vectors closer to the central weight vector. To do this, the DIM2 and DIM3 methods must be applied, and the derived dominance intensities are then weighted using the following expression:

$$
D I_{k l}^{*}=\frac{D I_{k l}}{d\left(w_{j}^{c}, w_{j}^{* l k}\right)} .
$$

The second extension is similar to the above in that weight vectors close to the central weight vector are given more importance. However, the weighting is now applied not to the dominance intensity values but to the pairwise dominance values in D. DIM2 and DIM3 are then applied as described before.

More recently, the same idea was extended concerning imprecision in DM preferences regarding both weights and component values/utilities. Instead of computing the pairwise dominance values $\left(D_{k l}\right)$, the method calculates:

$$
v_{k l}=\sum_{j=1}^{n} w_{j}^{c} v_{k j}^{c}-\sum_{j=1}^{n} w_{j}^{c} v_{l j}^{c},
$$

where $\left(w_{1}^{c}, \ldots, w_{n}^{c}\right)$ is the centroid or center of gravity of the polytope representing the weight space, and $\left(v_{k 1}^{c}, v_{l 1}^{c}\right), \ldots,\left(v_{k n}^{c}, v_{l n}^{c}\right)$ are the centroids or centers of gravity of the polytopes in the $n$ attributes delimited by the constraints accounting for alternatives $A_{k}$ and $A_{l}$. Note that the centroid is considered as the most representative point that verifies the constraints delimiting the polytope. Moreover, $D_{k l} \leq v_{k l} \leq-D_{l k}$.

The centroid of the polytope associated with constraints on component values in attribute $X_{j}$ for the alternatives $A_{k}$ and $A_{l}$ is

$$
\mathbf{v}_{j}^{c}=\left(v_{k j}^{c}, v_{l j}^{c}\right)=\frac{\int_{[0,1]^{2}} V_{j}^{k l} \mathrm{~d} v}{\int_{[0,1]^{2}} \mathrm{~d} v},
$$

where $V_{j}^{k l}$ is the set of constraints concerning component values in attribute $X_{j}$ for alternatives $A_{k}$ and $A_{l}$. Note that $V_{j}^{k l} \subset V_{j}$, which includes the constraints concerning component values in attribute $X_{j}$ for all the alternatives.

A technique for finding all the endpoints from a polytope delimited by constraints representing ordinal information is proposed in [2]. These endpoints can then be averaged to derive the centroid of the polytope.

As the representation of a constraint set as just a point would be an oversimplification, an interval centered on the central value is built as follows:

$$
I_{k l}=\left[I_{k l}^{L}, I_{k l}^{U}\right],
$$

where $I_{k l}^{L}=v_{k l}^{c}-m_{k l}$ and $I_{k l}^{U}=v_{k l}^{c}+m_{k l}$, and

$$
m_{k l}=\min \left\{\left(-D_{l k}-v_{k l}^{c}\right),\left(v_{k l}^{c}-D_{k l}\right)\right\} .
$$



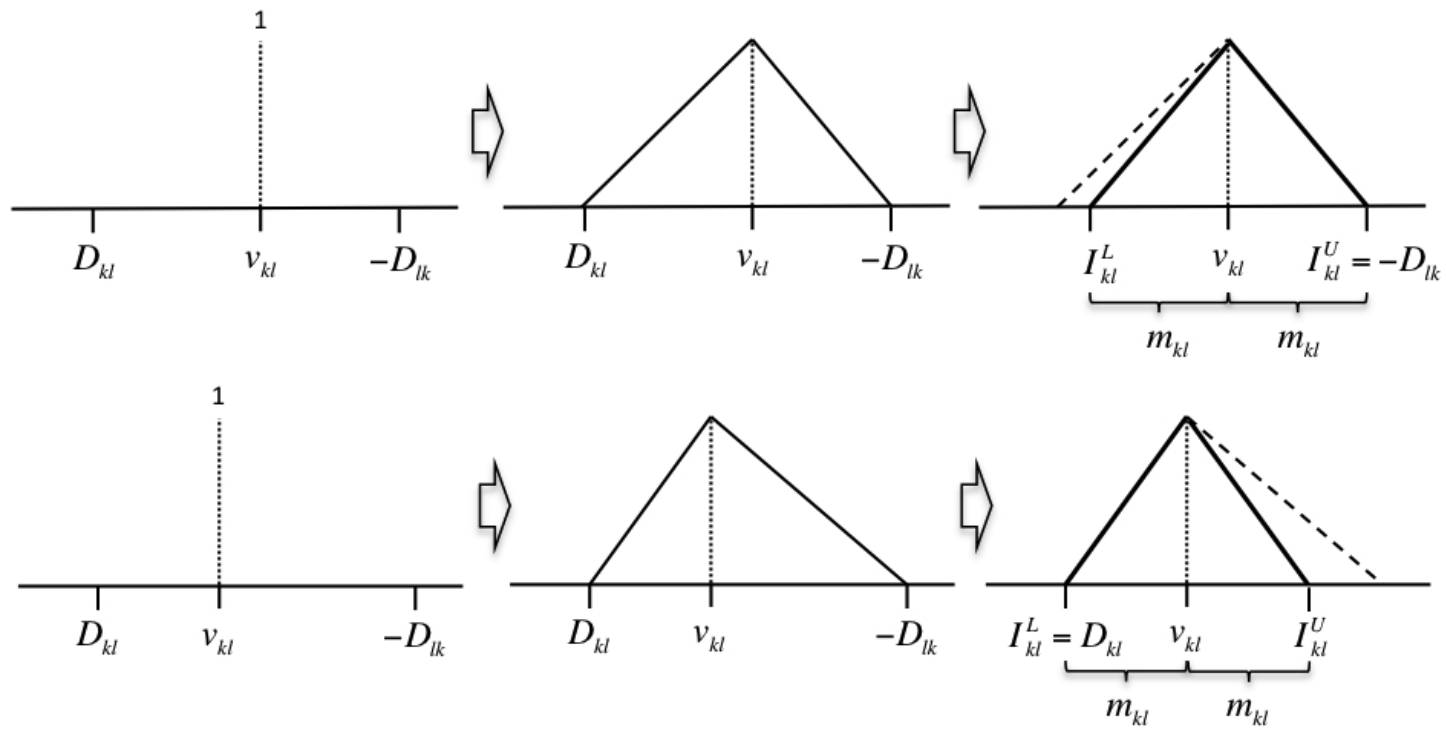

FiguRE 6. Building $\widetilde{I}_{k l}$.

Then the intervals $I_{k l}=\left[I_{k l}^{L}, I_{k l}^{U}\right]$ rather than $\left[D_{k l},-D_{l k}\right]$ are used to compute the dominance intensities in DIM3.

In [2] the method is applied to deal with ordinal information in both weights and component values. The DM provides an attribute importance ranking. Besides, the method takes into account a ranking of the alternatives in each attribute and also a ranking of the difference of values between consecutive alternatives, as in Figure 1. Rather than intervals, however, triangular fuzzy numbers are built:

$$
\widetilde{I}_{k l}=\left(I_{k l}^{L}, v_{k l}^{c}, I_{k l}^{U}\right)
$$

with the membership function (see Fig. 6).

$$
\mu_{\widetilde{I}_{k l}}(x)= \begin{cases}\frac{x-I_{k l}^{L}}{v_{k l}^{c}-I_{k l}^{L}}, & \text { if } I_{k l}^{L} \leq x \leq v_{k l}^{c} \\ 1, & \text { if } x=v_{k l}^{c} \\ \frac{x-I_{k l}^{U}}{v_{k l}^{c}-I_{k l}^{U}}, & \text { if } v_{k l}^{c} \leq x \leq I_{k l}^{U} \\ 0, & \text { otherwise. }\end{cases}
$$

Then the dominance intensities are computed as follows:

- If $v_{k l}^{c} \geq 0$, then $D I_{k l}=d\left(\widetilde{I}_{k l}, 0, f\right)$, where $d$ refers to Tran and Duckstein's distance [30], and $f$ is a weight function for differentiating risk-averse, risk-neutral or risk-prone DMs, as explained later.

- Else $\left(v_{k l}^{c}<0\right), D I_{k l}=-d\left(\widetilde{I}_{k l}, 0, f\right)$.

We compute a dominance intensity measure for each alternative $A_{k}, D I M_{k}=\sum_{l=1, l \neq k}^{m} D I_{k l}$, and rank alternatives according to $D I M_{k}$ values.

As mentioned above, the method is based on Tran and Duckstein's distance between fuzzy numbers, which can be expressed for the particular case of the distance from a triangular fuzzy number $\tilde{a}=\left(a_{1}, a_{2}, a_{3}\right)$ to a 
constant (specifically 0) by:

- If $f(\alpha)=\alpha$ (neutral), then

$$
d^{2}(\widetilde{a}, 0, f)=a_{2}^{2}+\frac{1}{3} a_{2}\left(a_{3}+a_{1}\right)+\frac{1}{18}\left[\left(a_{3}-a_{2}\right)^{2}+\left(a_{2}-a_{1}\right)^{2}\right]-\frac{1}{18}\left[\left(a_{2}-a_{1}\right)\left(a_{3}-a_{2}\right)\right] .
$$

- If $f(\alpha)=1$ (risk-prone), then

$$
d^{2}(\widetilde{a}, 0, f)=a_{2}^{2}+\frac{1}{2} a_{2}\left(a_{3}+a_{1}\right)+\frac{1}{9}\left[\left(a_{3}-a_{2}\right)^{2}+\left(a_{2}-a_{1}\right)^{2}\right]-\frac{1}{9}\left[\left(a_{2}-a_{1}\right)\left(a_{3}-a_{2}\right)\right] .
$$

- If $f(\alpha)=\alpha^{2}$ (risk-averse), then

$$
d^{2}(\widetilde{a}, 0, f)=a_{2}^{2}+\frac{1}{4} a_{2}\left(a_{3}+a_{1}\right)+\frac{1}{144}\left[\left(a_{3}-a_{2}\right)^{2}+\left(a_{2}-a_{1}\right)^{2}\right]-\frac{1}{96}\left[\left(a_{2}-a_{1}\right)\left(a_{3}-a_{2}\right)\right] .
$$

Note that $f(\alpha)$, which serves as a weight function, is a positive continuous function in $[0,1]$, and the distance is computed as a weighted sum of distances between two intervals along all of the $\alpha$-cuts from 0 to 1 .

Thanks to the presence of function $f$, DM participation is flexible. For example, when the DM is risk-neutral, $f(\alpha)=\alpha$ is a reasonable assumption. A risk-averse DM might want to attach more weight to information at a higher $\alpha$ level by using other functions, such as $f(\alpha)=\alpha^{2}$ or a higher power of $\alpha$. A constant $(f(\alpha)=1)$, or even a decreasing $f$ function, can be utilized for a risk-prone DM.

The results of Monte Carlo simulation techniques [2] demonstrate that the proposed method is clearly better than the $D M M s$ described above. Its performance is very similar to the method proposed by Sarabando and Dias [27], which was likewise developed to deal with ordinal information about DMs' preferences. Beside, Sarabando and Dias' method is less computationally demanding. However, it accounts only for ordinal information in both weights and component values, whereas $D M M s$ are applicable when imprecision concerning the DM's preferences and alternative performances are simultaneously represented in different ways, as demonstrated in the illustrative example introduced in the next section. Moreover, in the last extension of DIM the DM attitude toward risk can be incorporated to the analysis.

\section{An illustrative example}

We consider the selection of a supplier for cleaning services in a European public underground transportation company, where several conflicting criteria must be taken into account simultaneously, such as improving service levels and reducing total service costs.

The GMAA $[6,7,9]$ decision support system was used in [8] to deal with this selection problem based on decision analysis methodology. However, most of the attributes under consideration were subjective, and the DMs found it difficult to appraise the offers on their basis. Moreover, DMs also found it difficult to elicit the weights representing the relative importance of criteria by means of the trade-off weighting method $[7,10]$. However, they stated that they would feel more comfortable if ordinal information were allowed about the relative importance of criteria and about the appraisal of offers with respect to some attributes.

In this example, we allow DMs to use ordinal information both for attribute weights and for the appraisal of some offers with respect to some attributes. Moreover, rankings of the difference between the values of consecutive offers can be also taken into account for the different attributes.

First we built an objective hierarchy including all the relevant aspects related to the problem under consideration, aimed at improving DM understanding of the decision, see Figure 7.

There are five main top-level objectives: Delivery conditions and human resources (Delivery Con), which accounts for the level of consistency and coherence of the human resources allocated to the services; Technical merit and resources (Tech Resourc), which is an important efficiency factor leading to a significant reduction in labor cost; Price (Price), which represents the lowest price offered by suppliers; Quality control procedures 


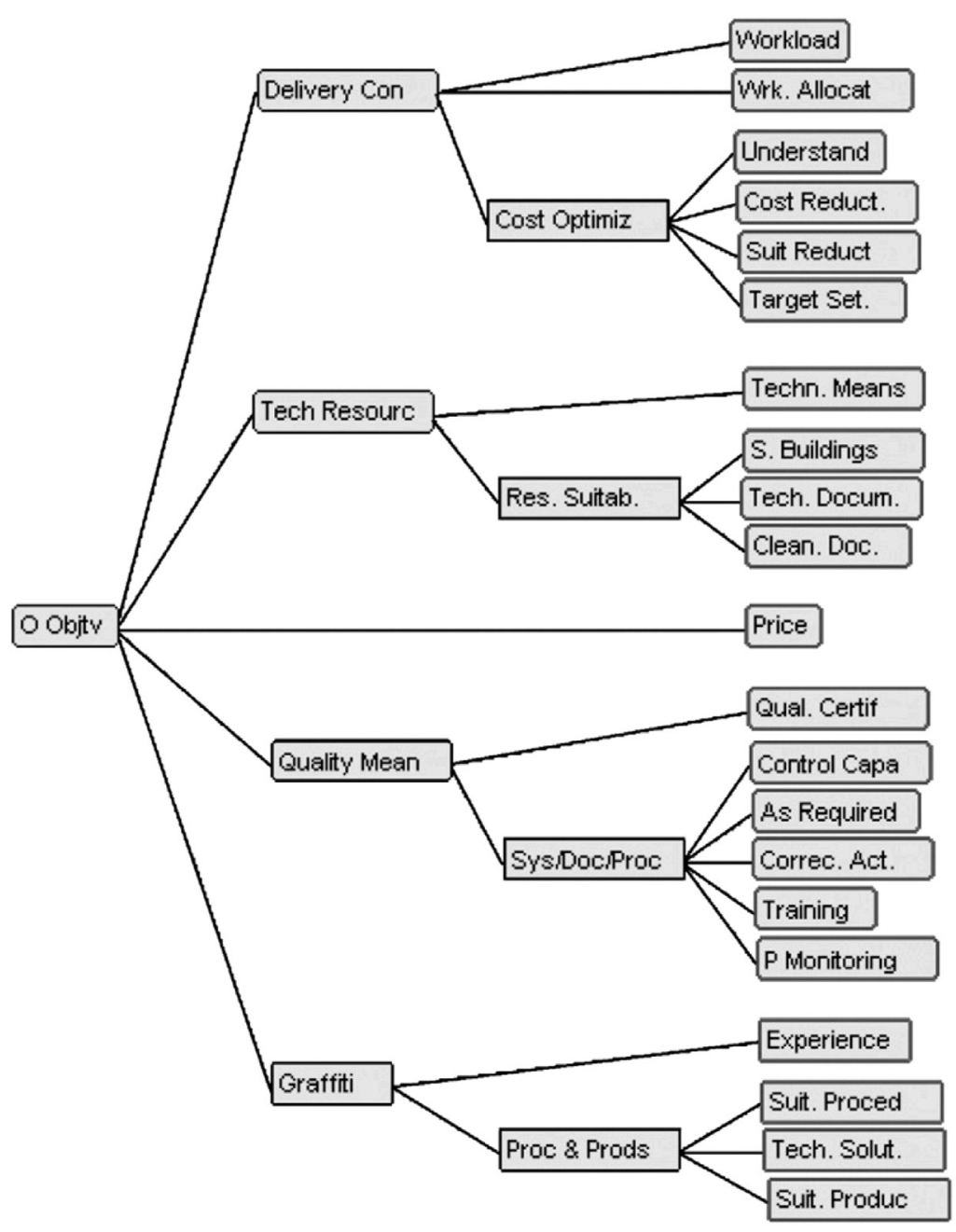

FIGURE 7. Objective hierarchy.

(Quality Mean), which accounts for accredited quality certifications and how quality systems and procedures are deployed; and Graffiti prevention and cleanup (Graffiti), which is one of the most common incidents detracting from the appearance of the underground buildings.

The Delivery conditions and human resources objective is split into three sub-objectives, Workload, Workload allocation and Cost optimization program. Workload (Workload) quantifies how much effort is deployed in terms of resources to achieve service performance objectives. Workload allocation (Wrk. Allocat) measures the coherence and consistency of the allocation of the resources throughout the underground facilities and buildings. Cost optimization program (Cost optimiz) measures the suitability of supplier cost reduction initiatives using two additional sub-objectives: Suitability of cost reduction initiatives (Suit Reduct), which quantifies how applicable and realistic the cost reduction initiatives are as things now stand and how they will ensure significant cost savings; and Cost reduction target setting and performance monitoring (Target Set), in which suppliers will be rewarded inasmuch as their offers include procedures and measures to successfully budget for and track cost savings.

Technical merit and resources (Techn. Resources) is also split into two sub-objectives: Quantity of technical means and Technical resources suitability. Suppliers will be rewarded inasmuch as their offers include large 
TABLE 1. Attribute with continuous scale.

\begin{tabular}{lcc}
\hline & Unit & Range \\
\hline$A_{1}:$ Workload & Absolute Percentage Error $(A P E)$ & {$[0,50]$} \\
$A_{2}:$ Wrk. Allocat & Mean Absolute Percentage Error $(M A P E)$ & {$[0,100]$} \\
$A_{5}:$ Techn. Means & Number of pieces of equipments & {$[0,90]$} \\
$A_{8}:$ Price & Monetary Units & {$[1500,3000]$} \\
\hline
\end{tabular}

amounts and extensive use of technical resources for Quantity of technical means (Techn. Means). Technical resources suitability (Res. Suitab.) is measured taking into account two additional sub-objectives: Equipment suitability to client's buildings (S. Buildings), which quantifies their appropriateness for underground facilities and possible adverse environmental impacts; and Cleaning product technical documentation (Clean. Doc.), which accounts for how well technical documentation describes the cleaning products.

Quality control procedures is measured in terms of two sub-objectives: Number of quality certifications (Qual. Certif) and Quality measuring systems and procedures (Sys/Doc/Proc). For the first, suppliers will be rewarded for having quality certifications accredited by national or European quality committees and institutions, whereas the second accounts for how quality systems and procedures are deployed.

This sub-objective is split into three sub-objectives: Supplier quality control capabilities (Control Capa.), which quantifies current supplier capabilities in terms of the supplier's quality assurance organization, its structure, number of resources, current skills and attested competencies; Poor performance corrective actions (Correc. Act.), which measures the proposed procedures and action plans to be put in place to solve poor performance and incidents when delivering cleaning services; and Quality performance monitoring (P. Monitoring), which accounts for the measuring system to be implemented, i.e., what will be inspected, how the inspection will be carried out and the measurement criteria to be used during inspection are evaluated to verify that they guarantee greater objectivity, completeness and high-quality services.

Finally, Graffiti prevention and cleanup takes into account two sub-objectives, past experiences and graffiti cleanup procedures and products. Past experiences in graffiti prevention and cleanup (Experience) verifies supplier competence, assessing details on how the supplier has successfully implemented these specific services for other clients. Graffiti cleanup procedures and product features (Proc and Prods) is measured by taking into account three additional sub-objectives: Suitability of graffiti cleanup procedures (Suit. Proced), which measures their appropriateness for underground facilities and possible adverse environmental impacts, Technical solution for prevention and cleanup (Tech. Solut) and Suitability of graffiti cleaning products (Suit. Produc), which quantify how well the technical documentation describes the equipment, leading to a clear identification and understanding of the equipment and product performance, respectively.

Next, attributes were established for the lowest-level objectives to indicate to what extent they were achieved by the respective offers. Table 1 shows the attribute names, units and ranges for four out of the 16 attributes under consideration measured on a continuous scale. Ordinal information about the component utilities of the offers was considered for the remaining attributes, as described later.

The units for $A_{1}$ : Workload and $A_{2}$ : Wrk. Allocat, i.e., APE and MAPE, represent the following:

- If the number of offers is odd and greater than 3, they are ordered from the highest to the lowest value, and a mean $(M)$ is assessed discarding the highest and lowest values.

- If the number of offers is even and greater than 2 , the mean is assessed considering all values. Then, we discard the offer whose difference with respect to the mean is the highest, positive or negative. A new mean $(M)$ is now assessed taking the remaining values.

- If the number of offers is 2 or 3 , the mean $(M)$ is assessed considering all values. 
TABLE 2. Offer performances.

\begin{tabular}{lcccccc}
\hline & Offer 1 & Offer 2 & Offer 3 & Offer 4 & Offer 5 & Offer 6 \\
\hline$A_{1}$ : Workload & 5.62 & 11.85 & 14.61 & 29.21 & 33.31 & 36.19 \\
$A_{2}$ : Wrk. Allocat & 13.63 & 30.66 & 19 & 5.78 & 16.27 & 26.6 \\
$A_{5}:$ Techn. Means & 23 & 19 & 63 & 31 & 80 & 24 \\
$A_{8}$ : Price & 1808.8 & 1932.9 & 1708.3 & 1799.4 & 1754.9 & 2199.3 \\
\hline
\end{tabular}

TABLE 3. Ordinal information concerning performances.

\begin{tabular}{|c|c|}
\hline & Ordinal information \\
\hline$A_{3}:$ Suit Reduct & Offer $3>$ Offer $5>$ Offer $2>$ Offer $1>$ Offer $4>$ Offer 6 \\
\hline$A_{4}:$ Target Set & Offer $3>$ Offer $1>$ Offer $4>$ Offer $2>$ Offer $6>$ Offer 5 \\
\hline$A_{6}:$ S. Buildings & Offer $3>$ Offer $2>$ Offer $4>$ Offer $6>$ Offer $1>$ Offer 5 \\
\hline$A_{7}:$ Clean Doc. & Offer $4>$ Offer $5>$ Offer $1>$ Offer $6>$ Offer $2>$ Offer 3 \\
\hline$A_{9}$ : Qual. Certif & Offer $1=$ Offer $2=$ Offer $3=$ Offer $4>$ Offer $5>$ Offer 6 \\
\hline$A_{10}$ : Control Capa & Offer $3>$ Offer $4>$ Offer $2>$ Offer $1>$ Offer $5>$ Offer 6 \\
\hline$A_{11}$ : Correct. Act. & Offer $4>$ Offer $5>$ Offer $3=$ Offer $1>$ Offer $2>$ Offer 6 \\
\hline$A_{12}:$ P. Monitoring & Offer $3=$ Offer $4>$ Offer $1>$ Offer $2>$ Offer $5>$ Offer 6 \\
\hline$A_{13}:$ Experience & Offer $3=$ Offer $4=$ Offer $5=$ Offer $6>$ Offer $2>$ Offer 1 \\
\hline$A_{14}$ : Suit. Proced & Offer $3>$ Offer $6>$ Offer $4>$ Offer $5>$ Offer $2>$ Offer 1 \\
\hline$A_{15}:$ Tech. Solut & Offer $3>$ Offer $4>$ Offer $6>$ Offer $5>$ Offer $2>$ Offer 1 \\
\hline$A_{16}:$ Suit. Produc & Offer $5>$ Offer $6>$ Offer $4>$ Offer $3>$ Offer $2>$ Offer 1 \\
\hline
\end{tabular}

Then, the absolute percentage error and the mean absolute percentage error for the $j$ th offer, $A P E_{j}$ and $M A P E_{j}$, are:

$$
A P E_{j}=\frac{A B S\left(M-\text { value }_{j}\right)}{M}, \quad M A P E_{j}=\frac{1}{N} \times \sum_{i=1}^{N} \frac{\left(M-\text { value }_{j}\right) \times 100}{M},
$$

where $N$ is the number of offers. Consequently, the best value for the attributes is 0 , while the worst values are 50 and 100 , respectively.

Six offers were considered for analysis. Table 2 shows the scores or performances of the different attributes using a continuous scale. Note that although the table reports precise performances, percentage deviations accounted for uncertainty about some performances. Specifically, a $20 \%$ and a $15 \%$ Workload deviation was introduced for Offers 5 and 6, and for Offer 4, respectively; a 10\% and a 20\% Wrk. Allocat deviation was introduced for Offers 3 and 6, and for Offer 2, respectively; and a 30\% and a 15\% Techn. Means deviation was introduced for Offers 3 and 5 , respectively.

Besides, Table 3 shows ordinal information concerning the appraisal of the six offers for the remaining attributes.

Moreover, rankings of the difference between the values of some consecutive offers were also taken into account by DMs for some attributes, see Table 4.

For instance, for $A_{16}$ : Suit. Produc, we have $\Delta_{16,5} \geq \Delta_{16,4} \geq\left\{\Delta_{16,1}, \Delta_{16,2}, \Delta_{16,3}\right\}$, where

$$
\begin{array}{ll}
\Delta_{16,1}=v_{16}(\text { Offer } 5)-v_{16}(\text { Offer } 6), & \Delta_{16,2}=v_{16}(\text { Offer6 })-v_{16}(\text { Offer } 4), \\
\Delta_{16,3}=v_{16}(\text { Offer } 4)-v_{16} \text { (Offer3), } & \Delta_{16,4}=v_{16}(\text { Offer3 })-v_{16}(\text { Offer } 2), \\
\left.\Delta_{16,5}=v_{16}(\text { Offer } 2)-v_{16} \text { (Offer }\right) . &
\end{array}
$$

This means that the difference in the appraisal of offers 2 and 1 is greater than the difference in the appraisal of offers 3 and 2, which is greater than the remaining differences between offers for that attribute. 
TABLE 4. Ordinal information about the difference between the values of consecutive offers.

\begin{tabular}{lc}
\hline & Ordinal information \\
\hline$A_{10}:$ Control Capa & $\Delta_{10,5} \geq\left\{\Delta_{10,1} \Delta_{10,2}, \Delta_{10,3}, \Delta_{10,4}\right\}$ \\
$A_{11}:$ Correct. Act. & $\Delta_{11,4} \geq\left\{\Delta_{11,1}, \Delta_{11,2}, \Delta_{11,3}, \Delta_{11,5}\right\}$ \\
$A_{12}:$ P. Monitoring & $\Delta_{12,2} \geq\left\{\Delta_{12,3}, \Delta_{12,4}, \Delta_{12,5}\right\}$ \\
$A_{13}:$ Experience & $\Delta_{13,4} \geq \Delta_{13,5}$ \\
$A_{14}:$ Suit. Proced & $\Delta_{14,5} \geq\left\{\Delta_{14,1}, \Delta_{14,2}, \Delta_{14,3}, \Delta_{14,4}\right\}$ \\
$A_{16}:$ Suit. Produc & $\Delta_{16,5} \geq \Delta_{16,4} \geq\left\{\Delta_{16,1}, \Delta_{16,2}, \Delta_{16,3}\right\}$ \\
\hline
\end{tabular}
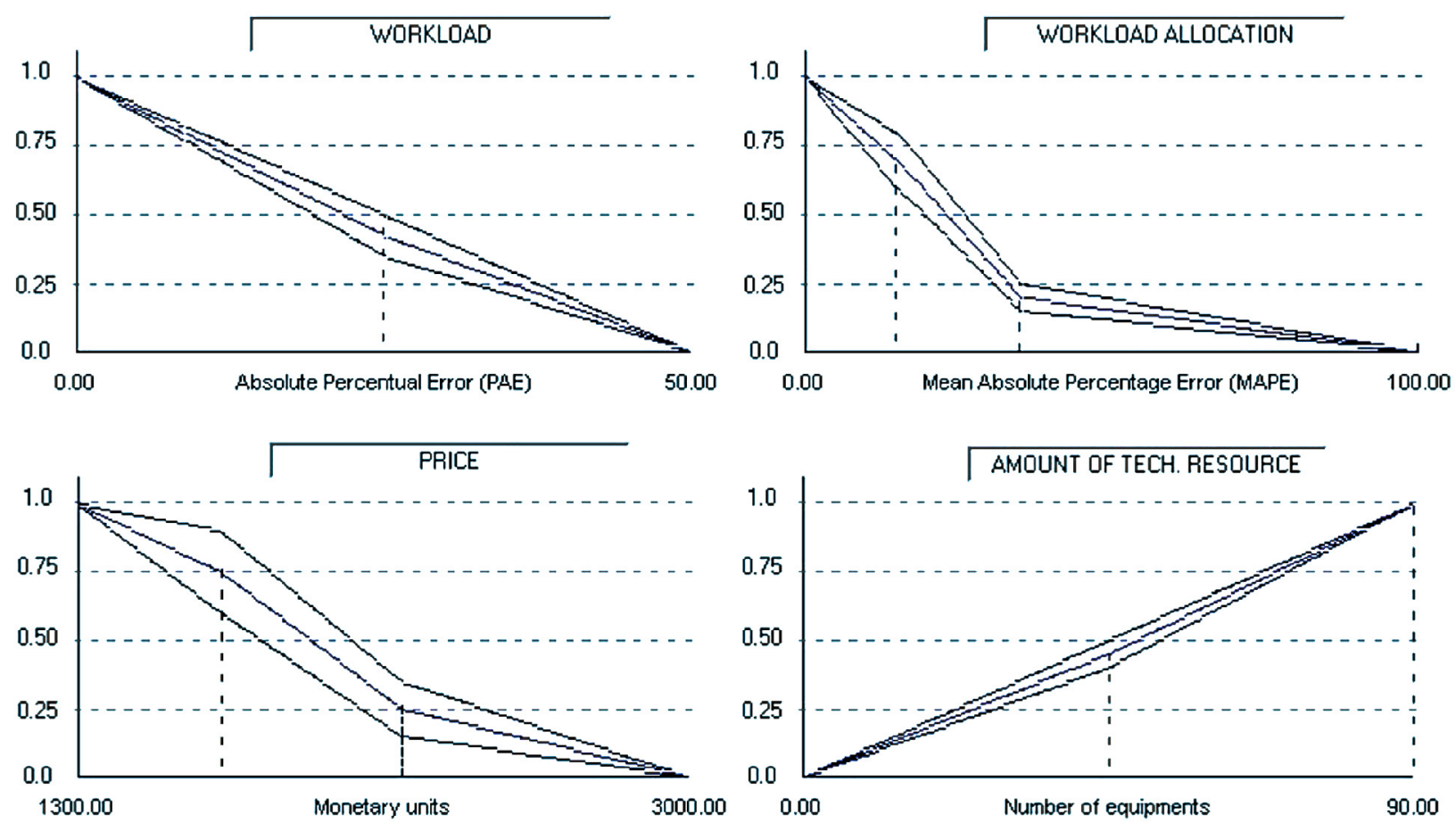

FiguRE 8. Imprecise component utility functions.

Regarding the quantification of the DMs preferences, Figure 8 shows the imprecise component utility functions assigned by the DMs to those attributes for which no ordinal information about the offer performances were provided. Note that utility functions are decreasing for $A_{1}$ : Workload, $A_{2}$ : Wrk. Allocat and $A_{11}$ : Price, and increasing for $A_{7}$ : Tech. Means.

Looking at the appearance of the component utility functions we realize that the DM's attitude toward risk is prone for the four attributes. Thus, we will consider this situation in Tran and Duckstein's distance when applying the extension of DIM3 with triangular fuzzy numbers to rank the offers.

As mentioned before, ordinal information was also used by DMs to represent the relative importance of the attributes as follows:

$$
w_{8}>3 w_{1}>w_{5}>w_{9}>w_{13}>w_{2}>\left\{w_{3}, w_{4}, w_{6}, w_{7}, w_{10}, w_{11}, w_{12}, w_{14}, w_{15}, w_{16}\right\},
$$


TABLE 5. Offer ranking.

\begin{tabular}{lccccc}
\hline & 1st & 2nd & 3th & 4th & 5 th \\
\hline Dominance intensity & 0.4921 & 0.0216 & -0.0997 & -0.1351 & -0.2789 \\
Ranking & Offer1 & Offer3 & Offer4 & Offer5 & Offer2 \\
\hline
\end{tabular}

i.e., the most important attribute for the decision is Price $\left(w_{8}\right)$, which is at least three times more important than Workload $\left(w_{1}\right)$. The next most important attributes are Tech. Means $\left(w_{5}\right)$, Qual. certif $\left(w_{9}\right)$, Experience $\left(w_{13}\right)$ and Wrk. Allocat $\left(w_{2}\right)$. Finally, Wrk. Allocat is more important than the remaining attributes.

Taking into account all the above information, the simplex method was used to solve the corresponding linear optimization problems to compute pairwise dominance values to derive the following dominance matrix:

$$
D=\left(\begin{array}{cccccc}
- & -0.0372 & -0.1373 & -0.0983 & -0.1566 & 0.1857 \\
-0.4249 & - & -0.3816 & -0.3141 & -0.2769 & 0.0125 \\
-0.3439 & -0.2244 & - & -0.2434 & -0.1986 & 0.0475 \\
-0.3741 & -0.2308 & -0.2603 & - & -0.3091 & 0.0392 \\
-0.4428 & -0.2532 & -0.3299 & -0.2866 & - & 0.0002 \\
-0.5763 & -0.4608 & -0.5322 & -0.5077 & -0.4447 & -
\end{array}\right) .
$$

Note that Offer 6 is dominated since all values in the last column are positive. Thus, it can be discarded for further analysis.

Table 5 shows the offer ranking derived from the last extension of DIM3 with triangular fuzzy numbers. Dominance measuring methods are the only methods that can be used for the incomplete information under consideration, i.e., performance intervals, ordinal information about the component values of some offers and rankings of the difference between the values of some consecutive offers, and ordinal information about weights. Note that the extension of DIM3 with triangular fuzzy numbers accounts for DM's attitude toward risk.

Note that Offer 1 is best ranked, followed by offers 3, 4, 5 and 2. The three best ranked offers are the same than in the ranking reported in [8], in which the uncertainty about the offer performances and the imprecision concerning weights were both represented by intervals.

\section{Conclusion}

Dominance measuring methods $(D M M s)$ have been shown to be a valid approach for dealing with complex decision-making problems with imprecise, incomplete or partial information within multi-attribute value/utility theory.

We have reviewed different $D M M s$ for dealing with imprecise information about decision-maker preferences, identifying drawbacks associated with the original $D M M s$ and describing other DMMs aimed at overcoming these shortcomings. The performance of these methods has been compared in the literature with other existing approaches on the basis of two efficiency measures (the hit ratio and the rank-order correlation.

The results of the applied Monte Carlo simulation techniques demonstrate that DIM2 outperforms AP1, $A P 2$ and the adapted classical decision rules when both intervals and ordinal information about weights are considered. Besides, SMAA-2 slightly outperformed DIM2 when intervals were considered, and there was no significant difference between DIM2 and the ROC method when ordinal information was considered (DIM2 outperformed the other surrogate weighting methods).

However, $R O C$ and $S M A A-2$ are only applicable when ordinal information about weights is considered and $D M M s$ are more general since other linear restrictions could be considered to derive the dominance matrix. 
Besides, the last extension of DIM3 with triangular fuzzy numbers outperforms previous $D M M s$, and there is no significant difference between DIM3 and the method proposed by Sarabando and Dias when dealing with ordinal information concerning both weights and component values.

However, Sarabando and Dias account only for ordinal information regarding both weights and component values, whereas $D M M$ is applicable when imprecision concerning DM preferences and alternative performances are simultaneously represented in different ways and the DM's attitude toward risk can be incorporated to the analysis, as demonstrated in the illustrative example.

\section{REFERENCES}

[1] E.A. Aguayo, Dominance Intensity Methods for Ranking of Alternatives in MCDM with Imprecise Information. Ph.D. thesis, Universidad Politécnica de Madrid, Madrid (2014).

[2] E.A. Aguayo, A. Mateos and A. Jiménez-Martín, A new dominance intensity method to deal with ordinal information about a DM's preferences within MAVT. Knowl. Based Syst. 69 (2014) 159-169.

[3] B.S. Ahn and K.S. Park, Comparing methods for multiattribute decision making with ordinal weights. Comput. Oper. Res. 35 (2008) 1660-1670.

[4] F. Barron and B. Barret, Decision quality using ranked attribute weights. Man. Sci. 42 (1996) 1515-1523.

[5] Y. Eum, K. Park and H. Kim, Establishing dominance and potential optimality in multi-criteria analysis with imprecise weights and value. Comput. Oper. Res. 28 (2001) 397-409.

[6] A. Jiménez, S. Ríos-Insua and A. Mateos, A decision support system for multiattribute utility evaluation based on imprecise assignments. Decis. Support Syst. 36 (2003) 65-79.

[7] A. Jiménez, S. Ríos-Insua and A. Mateos, A generic multi-attribute analysis system. Comput. Oper. Res. 33 (2006) $1081-1101$.

[8] A. Jiménez, A. Mateos, S. Ríos-Insua and L.C. Rodríguez, Contracting of cleaning services in a European underground transportation company with the aid of a DSS. Decis. Support Syst. 43 (2007) 1485-1498.

[9] A. Jiménez, M.C. Suárez-Figueroa, A. Mateos, A. Gómez-Pérez, M. Fernández-López, A MAUT approach for reusing domain ontologies on the basis of the NeOn methodology. Int. J. Info. Tech. Decis. Mak. 12 (2013) 945-968.

[10] R.L. Keeney and H. Raiffa, Decision with Multiple Objectives: Preferences and Value-Tradeoffs. Wiley, New York (1976).

[11] R. Lahdelma and P. Salminen, SMAA-2: Stochastic multicriteria acceptability analysis for group decision making. Oper. Res. 49 (2001) 444-454.

[12] R. Lahdelma, J. Hokkanen and P. Salminen, SMAA-Stochastic multiobjective acceptability analysis. Eur. J. Oper. Res. 106 (1998) 137-143.

[13] R. Lahdelma, K. Miettinen and P. Salminen, Ordinal criteria in stochastic multi-criteria acceptability analysis (SMAA). Eur. J. Oper. Res 147 (2003) 117-127.

[14] K. Lee, K. Park and S. Kim, Dominance, potential optimality, imprecise information and hierarchical structure in multi-criteria analysis. Comput. Oper. Res. 29 (2002) 1267-81.

[15] A. Mateos, S. Ríos-Insua and A. Jiménez, Dominance, potential optimality and alternative ranking in imprecise decision making. J. Oper. Res. Soc. 58 (2007) 326-336.

[16] A. Mateos, A. Jiménez and J.F., Blanco, Ranking methods based on dominance measures accounting for imprecision, edited by F. Rossi and A. Tsoukis. Vol. 5783 of LNAI. Springer, Berlin (2009) 328-339.

[17] A. Mateos, A. Jiménez, E.A., Aguayo and A. Moreno, Ranking alternatives on the basis of a dominance intensity measure. Proc. of 25th IFIP WG 8.3 Int. Conf. Decision Support Systems (2010).

[18] A. Mateos, A. Jiménez and J.F. Blanco, Measuring method performance under incomplete information about weights. J. Multi-Crit. Decis. Anal. 19 (2012) 129-142.

[19] A. Mateos, A. Jiménez, E.A. Aguayo and P. Sabio, Preference intensity in MCDM when and additive utility function represents DM preferences. In Uncertainty Modelling in Knowledge Engineering and Decision Making. Edited by C. Kahraman, E.E. Kerre and P.T. Bozbura. New Jersey, World Scientific (2012) 106-111.

[20] A. Mateos, A. Jiménez-Martín, E.A. Aguayo and P. Sabio, Dominance intensity measuring methods in MCDM with ordinal relations regarding weights. Knowl. Based Syst. 70 (2014) 26-32.

[21] K. Park, Mathematical programming models for characterizing dominance and potential optimality when multicriteria alternative values and weights are simultaneously incomplete. IEEE Trans. Syst., Man. Cyb. Part A 34 (2004) 601-14.

[22] J. Puerto, A.M. Mármol, L. Monroy and F.R. Fernández, Decision criteria with partial information. IEEE Trans. Oper. Res. 7 (2000) 51-65.

[23] H. Raiffa, The Art and Science of Negotiation. Cambridge: Harvard University Press (1982).

[24] D. Ríos Insua, Sensitivity Analysis in Multi-objective Decision Making. Springer, New York (1990).

[25] D. Ríos and S. French, A framework for sensitivity analysis in discrete multiobjective decision-making. Eur. J. Oper. Res. 54 (1991) 176-190. 
[26] A. Salo and R.P. Hamalainen, Preference ratio in multiattribute evaluation (PRIME)-elicitation and decision procedures under incomplete information. IEEE Trans. Syst., Man. Cyb. Part A 31 (2001) 533-545.

[27] P. Sarabando and L.C. Dias, Multi-attribute choice with ordinal information: a comparison of different decision rules. IEEE Trans. Syst., Man. Cyb. Part A 39 (2009) 545-554.

[28] P. Sarabando and L.C. Dias, Simple procedures of choice in multicriteria problems without precise information about the alternatives values. Comput. Oper. Res. 37 (2010) 2239-2247.

[29] T.J. Stewart, Robustness of additive value function method in MCDM. J. Multi-Crit. Decis. Anal. 5 (1996) 301-309.

[30] L. Tran and L. Duckstein, Comparison of fuzzy numbers using a fuzzy number measure. Fuzzy Sets Syst. 130 (2002) 331-341.

[31] W.G. Stillwell, D.A. Seaver and W.A. Edwards, Comparison of weight approximation techniques in multiattribute utility decision making. Org. Behav. Human Decis. Proc. 28 (1981) 62-77.

[32] M. Weber, Decision making with incomplete information. Eur. J. Oper. Res. 28 (1987) 44-57.

[33] R.L. Winkler and W.L. Hays, Statistics: Probability, Inference and Decision. Holt, Rinehart \& Winston, New York (1985). 